\title{
Confirmation of Hypothesis on Cable Properties for Cable-driven Robots
}

\author{
J. Alexandre dit Sandretto ${ }^{1}$, G. Trombettoni ${ }^{12}$ and D. Daney ${ }^{1}$ \\ ${ }^{1}$ Coprin INRIA, France e-mail: Julien.Alexandre_dit_Sandretto@inria.fr \\ ${ }^{2}$ I3S, France e-mail: Gilles.Trombettoni@inria.fr
}

\begin{abstract}
In the cable-driven robot studies, the mass and the elasticity of cables are often neglected, particularly for small-sized robots. Indeed, this assumption is interesting to simplify the robot model, and consequently it is used in control, design or calibration. We propose in this paper a method using Interval Analysis to judge the validity of this hypothesis in a given workspace, according to the cable characteristics, i.e., the applied tensions and the robot configuration.
\end{abstract}

Key words: Cable-driven robot, Interval analysis

\section{Introduction}

Cable-driven robots have several interesting properties such as a reduced mass of mobile parts (i.e. cables have a mass negligible compared to the load), and a potentially large workspace. They are used in several applications, including a flying camera system [1], heavy load transportation, contour crafting [3].

These robots are structurally similar to classical parallel manipulators, but are driven by cables instead of rigid links. The past studies performed have shown the complexity of kinematics for parallel robots [10]. In addition, the unilateral driving property of cables leads to hardly solvable kinematics and dynamics behavior due to the flexibility, mass and elasticity of the cables.

Several studies have been achieved on cable-driven robot kinematics [11], workspace [5] or calibration [14]. However, a majority of these researches on cabledriven robots use a fundamental hypothesis: the cables driving the platform are mass-less and non-elastic. This hypothesis leads to a simplification which permits control, kinematics, calibration, design, as a classical redundant Gough platform for example. In this paper, we propose a way to verify the validity of this hypothesis in the whole workspace of a given robot.

In robotics, especially when the handled problem is complex, several pioneering researchers have used the capabilities of interval methods. For example, Jaulin et al. showed in their book how to identify parameters [8]; Merlet solved the forward 
kinematics of a Gough platform [9]; Chablat designed 3 d.o.f parallel machines [4] and Pott and Hiller performed an optimization for parallel machines [2].

In these works, interval analysis is used because it can certify properties, which is sometimes crucial in robotics. Indeed, interval analysis $[8,12,13]$ can handle the whole continuous space, contrarily to approaches based on discretization that may lose some solutions.

\section{Cable-driven robots}

The goal of our national project, named CoGiRo, is to build a giant parallel cabledriven robot. This raises numerous issues: design, mechanical conception, modeling, vision-based control, etc. We will focus on the kinematics, static is only used for the cable model (not for the robot equilibrium) and we do not deal with the dynamic model.

\subsection{Robot description}

A parallel cable-driven robot is made of a mobile platform (end-effector) connected to a fixed base by $m$ cables. These cables can vary in length by the actuation of $m$ pulleys linked to $m$ rotary engines. The variation in length and tension of cables generates a movement in $n$ degrees of freedom (position and/or orientation). In the sketch presented in Fig. 1, the mobile platform (mobile reference frame $\Omega_{C}$ )

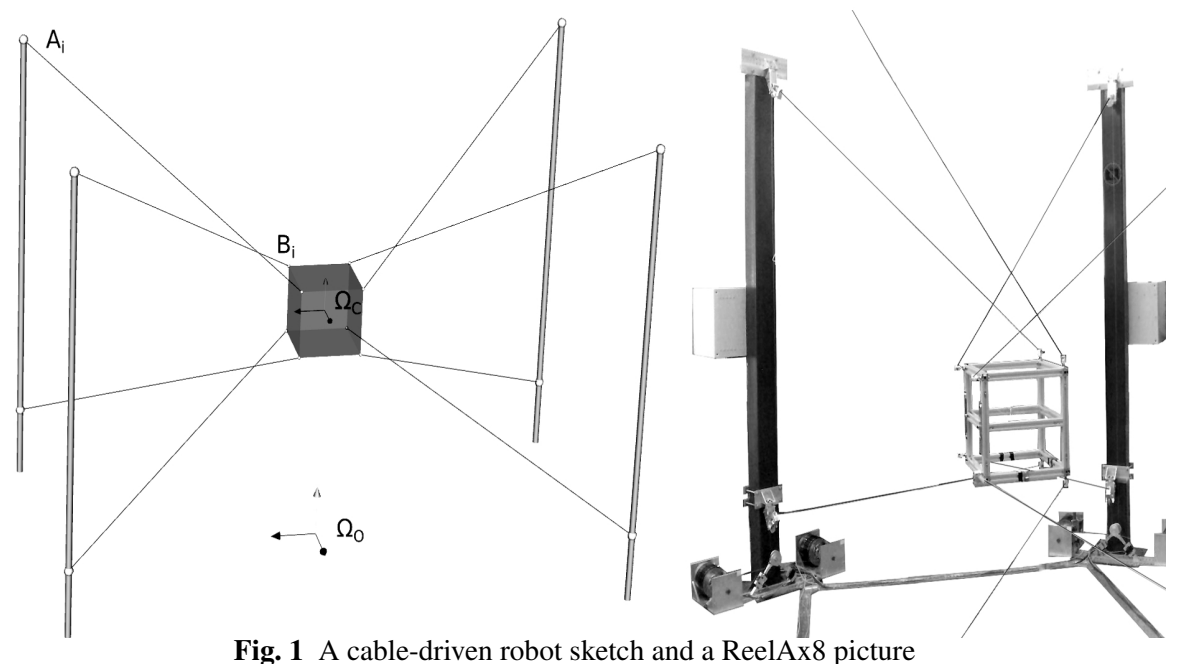

Fig. 1 A cable-driven robot sketch and a ReelAx8 picture

is connected to the base (fixed reference frame $\Omega_{O}$ ) by $m=8$ cables ( $m>n$ to be fully controllable [11]). The $i^{t h}$ cable connects the point $A_{i}$ of the base (coordinate 
$a_{i}$ in $\Omega_{O}$ ) to the point $B_{i}$ on the mobile platform (coordinate $b_{i}$ in $\Omega_{C}$ ). The pose of the mobile $X=(P, R)$ (defined by the position $P$ and the orientation matrix $R$ of $\Omega_{C}$ w.r.t. $\Omega_{O}$ ) is directly controlled by the length and the tension in each cable. The workspace $W_{X}$ is the set of all possible couples $(P, R)$ for the robot.

The prototype, named ReelAx8 and shown in Fig. 1, was built by the TECNALIA company (www.tecnalia.com) in collaboration with the LIRMM laboratory (www.lirmm.fr). Eight cables, wound on winches, are attached by spherical joints to the eight corners of a cube shaped platform of about 40 centimeters large. Four pairs of winches are fixed on posts up to three meters arranged at the four corners of a 3 meters by 4 meters rectangle. The prototype is given with a rectangular and centered workspace (see Fig. 3) of 2 by 1 meter on floor, 1 meter high and \pm 5 degrees of rotation on each axis: $W_{X}=\{X=[P, R], P \in[1,3] \times[1,2] \times[1,2], R \in$ $[-5,5] \times[-5,5] \times[-5,5]\}$.

\subsection{Cable model}

Cable-driven robots take advantage of the use of cables, allowing large workspace, light actuators in comparison to the possible load mass, and low cost. However, cable-driven robots suffer from the complex kinematics and dynamics of cables.

A well-known realistic model that is often used for the kinematics of cables is proposed by Irvine in [7]. In the Irvine model, the length of a cable depends on its tension. It is given for one cable and the equations are expressed in a plane made of the points $\bar{A}$ and $\bar{B}$ and the gravitational force.

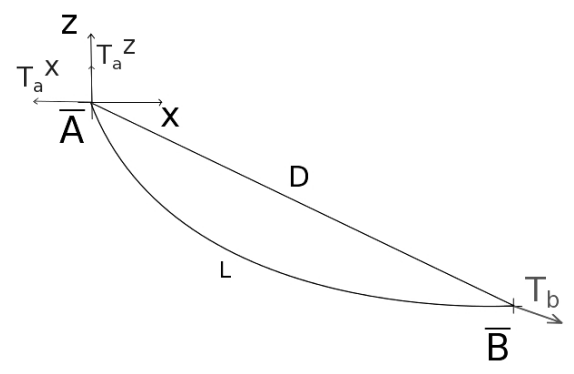

Fig. 2 A cable in a plane $\{\bar{A}, \bar{B}, \vec{P}\}$

The Irvine model considers the geometric and static parameters of the configuration and the cable properties: attachment points $\bar{A}$ (on base) and $\bar{B}$ (on platform); linear mass $m$, tightness $k$ and length $L$ of the cable; applied tensions $T_{b}$ in $\bar{B}$ and $T_{a}$ in $\bar{A}$. The system of three equations to be solved in order to obtain the actual length of cable and the tension distribution on point $\bar{A}=[0,0]$ is: 


$$
\left\{\begin{array}{lr}
\bar{B}_{x}= & \frac{T_{a}^{x} L}{k}+\frac{\left|T_{a}^{x}\right|}{m g}\left[\sinh ^{-1}\left(\frac{T_{a}^{z}}{T_{a}^{x}}\right)-\sinh ^{-1}\left(\frac{T_{a}^{z}-m g L}{T_{a}^{x}}\right)\right] \\
\bar{B}_{z}= & \frac{m g L^{2}}{k}\left(\frac{T_{a}^{z}}{m g L}-\frac{1}{2}\right)+\frac{1}{m g}\left[\sqrt{T_{a}^{x 2}+T_{a}^{z 2}}-\sqrt{T_{a}^{x 2}+\left(T_{a}^{z}-m g L\right)^{2}}\right] \\
T_{b}= & \sqrt{T_{a}^{x^{2}}+\left(T_{a}^{z}-m g L\right)^{2}}
\end{array}\right.
$$

The fact that a robot is controlled with cables, which have complex kinematics, leads to some problems in the classical fields of robotics:

- complex modeling, control and design;

- unworkable existing methods for the workspace determination;

- unfeasible self-calibration.

The hypothesis of non-elastic and mass-less cables is very useful to simplify the modeling. Moreover, this hypothesis is often realistic and generate a negligible error in robot accuracy. The majority of papers dealing with these subjects use this hypothesis, and replace the real length of cables $L$ (depending on tensions) by the distances $D=A B$. Under this assumption, the model is highly simplified.

\section{Checking of non-elasticity and mass-less hypothesis}

The hypothesis of non-elasticity and mass-less done on cables properties has to be checked on one cable before any static or dynamic modeling which are currently not mastered by the community. Our problematics is therefore to verify this hypothesis in the whole workspace of the robot $W_{X}$ to bring the guarantee that the simplification is valid.

For this purpose, we compute the errors $\sigma_{i}=\left|L_{i}-D_{i}\right|$ made between the length $L_{i}$ given by Irvine's model -function of $B_{i}, T_{B_{i}}$ and the cable parameters- and the distance $D_{i}$, only function of $B_{i} . B_{i}$ itself function of $X \in W_{X}$. We then verify that these errors all lie under an acceptable threshold $\varepsilon$ (which could be selected in function of the expected articular accuracy): $\sigma_{i} \leq \varepsilon, i=1 . . m$. The coordinates of $B_{i}$ in $\Omega_{O}$ are $e_{i}=P+R . b_{i}, b_{i}$ being the coordinates of $B_{i}$ expressed in the platform reference $\Omega_{C}$ (defined by the platform geometry). The hypothesis is verified on a pose $X \in W_{X}$, if for the every $m$ points $e_{i}: \sigma_{i} \leq \varepsilon$.

Therefore, we can define the subset $S_{X}$ of acceptable poses as follows: $S_{X}=\left\{X \in W_{X}, \forall i \in 1 . . m: \sigma_{i} \leq \varepsilon\right\}$. The proposed verification consists in checking the hypothesis in all the poses of the workspace. A sufficient condition is based on the dual set $S_{! X}=\left\{X \in W_{X}, \exists i \in 1 . . m: \sigma_{i}>\varepsilon\right\}$. Interval methods can determine if $S_{! X}=\emptyset$, which implies the hypothesis holds on $W_{X}$.

Moreover, the $m$ points $B_{i}$ depending on $X$ all belong to the same parallelepiped, whatever can be $X \in W_{X}$. Thus, we are satisfied with testing the hypothesis for only one cable. In addition, the parallelepiped built with the $e_{i}$ is entirely covered by the diagonal plane with a simple rotation around the $z$ axis. Overall, the study of the workspace $W_{X}$ can be reduced to the one of the diagonal plane $W_{B}$, as shown 


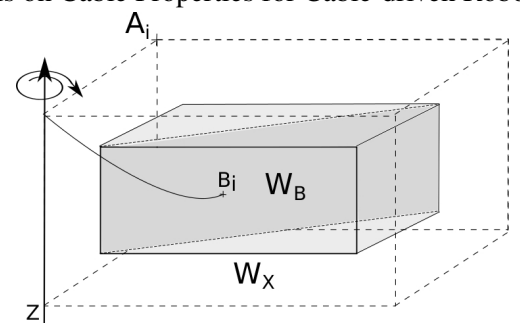

Fig. 3 Robot space frame in dashed lines, workspace $W_{X}$ and diagonal plane $W_{B}$

in Fig. 3. The point $B \in W_{B}$ could be expressed in the plane reference frame like in Irvine's model definition: $B=\left[\bar{B}_{x}, \bar{B}_{z}\right]$. With this simplification, we define the sub-space of point $B$ where the hypothesis is valid: $S_{B}=\left\{B \in W_{B}: \sigma \leq \varepsilon\right\}$. And we will introduce the complementary: $S_{! B}=\left\{B \in W_{B}: \sigma>\varepsilon\right\}$. We remark that $S_{B} \cup S_{! B} \equiv W_{B}$. By construction of the simplification, if a solution is found in $S_{! B}$, a solution exists in $S_{! X}$ and the hypothesis is not valid in the whole workspace. It's also easier to find zero solution in $S_{! B}$ that prove that $S_{B} \equiv W_{B}$. Proving that $S_{! B}$ has no solution implies indeed that the hypothesis $\sigma \leq \varepsilon$ is verified for every point in the workspace.

\section{Interval methods for constraint satisfaction and optimization}

This problematics demands a robust solver which could consider a whole space made of an infinity of points and give a reliable result. Interval analysis meets this requirement by using algorithmic principles exploiting constraints and sub-spaces containing an infinity of points, without risk of solution loss.

An interval $\left[x_{i}\right]=\left[\underline{x_{i}}, \overline{x_{i}}\right]$ defines the set of reals $x_{i}$ s.t. $x_{i} \leq x_{i} \leq \overline{x_{i}}$. A box $[x]$ is the Cartesian product of intervals $\left[x_{1}\right] \times \ldots \times\left[x_{i}\right] \times \ldots \times\left[x_{n}\right]$. Its width is defined by $\max _{i} w\left(\left[x_{i}\right]\right)$.

Interval methods for solving a constraint system

Interval methods can accurately approximate by boxes the set of solutions of a constraint system. The solving process starts from an initial box representing the search space and builds a search tree, following a Branch \& Contract scheme:

- Branch: the current box is bisected on one dimension (variable), generating two sub-boxes.

- Contract: filtering (also called contraction) algorithms reduce the bounds of the box with no loss of solution.

The process terminates with atomic boxes of size at most $\varepsilon$ on every dimension. Contraction algorithms comprise interval Newton-like algorithms issued from the numerical interval analysis community [12] along with algorithms from constraint programming.

Constrained optimization 
Interval methods can also deal with a more difficult problem, constrained optimization, in which a solution must be found that minimizes an objective function while satisfying the set of constraints. To do so, the strategy follows a branch and bound schema [6]. At each iteration, the algorithm selects in the list a box $[x]$. It chooses a branching variable $x_{i} \in x$ heuristically, bisects $\left[x_{i}\right]$ and applies the main Contract\&Bound procedure on the two sub-boxes. In addition to the contraction phase mentioned above, the procedure Contract \& Bound resorts to a lower bounding phase computing a cost $l b$ and an upper bounding phase computing a cost $u b$ [6].

The search terminates when $u b-l b$ reaches a precision $\varepsilon_{o b j}$.

\section{Experimentation}

The solving is performed by adapting an interval tool developed in the COPRIN team and briefly described in Section 4.

\section{Case 1: existing prototype}

The cables used have the following characteristics: $k=137 \mathrm{kN} / \mathrm{m}, m=0.007 \mathrm{~kg} / \mathrm{m}$. With the workspace $W_{X}$ introduced in the description of the robot, the plane $W_{B}$ to be tested is the diagonal plane of the rectangular parallelepiped $[1,3] \times[1,2] \times[1,2]$ and $W_{B}=[1,3.7] \times[1,2]$. We fix $\varepsilon=0.005 m \simeq$ expected accuracy of robot.

The sensors give, during our tests, a minimal tension of $20 \mathrm{~N}$ and a maximal one of $120 N$. So $20 \leq T_{b} \leq 120$.

\section{Case 2: robot under construction}

We consider the same architecture robot but with heavier cables and larger workspace. The cables are in the same steel with a tightness $k=137 \mathrm{kN} / \mathrm{m}$, and a lineic mass $m=0.092 \mathrm{~kg} / \mathrm{m}$. The workspace is estimated at $W_{B}=[1,8] *[1,10]$ for the next prototype for which we also expect an accuracy of $1 \mathrm{~cm}$. The tension should be between $40 N$ (without load) and $1000 N$ (at maximal load).

\subsection{Hypothesis confirmation}

We compute an overestimation of the subset $S_{! B}$, noted $\square S_{! B}$, by using the constraint system made with (1) and the additional constraint $\sigma>\varepsilon$. If no solution is found in $\square S_{! B}$, no solution exists in $S_{! B}$, and the hypothesis is valid in the considered workspace.

Case 1: No solution is found by our tool, therefore the hypothesis is acceptable for the studied robot. The model using the simplification is thus sufficiently accurate. The solving process achieved in the whole workspace take about 2 hours. For a reduced workspace, for example one by one meter, the resolution is performed in about 10 minutes.

Case 2: A solution for $\square S_{! B}$ is immediately found ( $\approx 1$ second). The hypothesis seems therefore too strong and a more complex model must be developed for the 
giant robot under construction. Otherwise, the robot model accuracy could be highly deteriorated.

\subsection{Quantifying the error}

In addition to the yes/no results obtained about the hypothesis validation, global optimization gives the opportunity to enrich the knowledge about the robot. However, we could expect additional quantified information such as:

- The minimal tension satisfying the hypothesis, defined by $S_{T_{b}}=\operatorname{Min}_{T_{b}}, \forall B \in S_{B}$

- The maximal error committed in the workspace, defined by $S_{\sigma}=M a x_{\sigma}, \forall B \in$ $W_{B}, \forall T_{b} \in\left[T_{\min }, T_{\max }\right]$

Case 1: The analysis of the prototype model (for which the hypothesis has been proved acceptable by our verification method) provides useful information gathered in Table 1

- Minimal $T_{b}$ to keep $|\sigma|<\varepsilon$, see Table1, column 1;

- Maximal $|\sigma|$ for $T_{b}=20 N$, see Table1, column 2;

- Maximal $|\sigma|$ for $T_{b}=120 N$, see Table1, column 3;

- Maximal $T_{b}$ to keep $|\sigma|<\varepsilon$, see Table1, column 4;

The values found confirm the hypothesis validation.

Case 2: The same model analysis protocol is followed for the cable-driven robot under construction and the results are presented in Table 1.

- Maximal $|\sigma|$ for $T_{b}=40 N$, see Table1, column 5;

- Minimal $T_{b}$ to keep $|\sigma|<\varepsilon$, see Table1, column 6;

- Maximal $T_{b}$ to keep $|\sigma|<\varepsilon$, see Table1, column 7;

- Maximal $|\sigma|$ for $T_{b}=1000 N$, see Table1, column 8;

The values found confirm the hypothesis rejection, even if the lower tension bound is close to the minimal tension for which the hypothesis is valid.

\begin{tabular}{|l||l|l|l|l||l|l|l|l|}
\hline & 1 & 2 & 3 & 4 & 5 & 6 & 7 & 8 \\
\hline Error $\sigma(\mathrm{m})$ & 0.005 & $\mathbf{0 . 0 0 0 6}$ & $\mathbf{- 0 . 0 0 3 7}$ & -0.005 & $\mathbf{0 . 0 1 0 0 9}$ & 0.01 & -0.01 & $\mathbf{- 0 . 0 9 3}$ \\
\hline Tension $T_{b}(\mathrm{~N})$ & $\mathbf{1 . 4}$ & 20 & 120 & $\mathbf{1 7 1 . 5}$ & 40 & $\mathbf{4 0 . 1}$ & $\mathbf{1 2 1}$ & 1000 \\
\hline Solv. time $(\mathrm{s})$ & 200 & 3 & 5 & 30 & 72 & 3 & 6000 & 5 \\
\hline
\end{tabular}

Table 1 Results (in bold) obtained by optimization processes

\section{Conclusion}

In our research, we have done the hypothesis of mass-less and non-elasticity of cables in order to self-calibrate the robot presented in Section 2. Indeed, to self- 
calibrate a cable-driven robot, we must consider it as a redundantly actuated manipulator. This redundancy is obtained with the simplified model under non-elasticity and mass-less assumption. This hypothesis is validated with our method, the simplification is thus acceptable and this robot is self-calibratable. In the robot under construction, the hypothesis is rejected. To self-calibrate this giant crane, we must find a sub-workspace where the hypothesis is acceptable or use a more complex model.

To conclude, we have designed an operational tool for analyzing the difference between a real cable model and a strong simplification of it. The method described in this paper has provided interesting and useful results for our study of cable-driven robots. Its implementation represents a first software version of a dedicated design tool which could be incorporated in an "Appropriate design" approach.

Finally, this dedicated tool can be useful for modeling, designing and optimizing in a reliable way robots, but also other mechanisms that make use of cables.

\section{Acknowledgment}

This work was supported in part by the French National Research Agency (ANR) under grant 2009-SEGI-018 (CoGiRo ANR project) and in part by the Région Languedoc-Roussillon under grant 115217.

\section{References}

1. Skycam, 2007

2. Manfred Hiller Andreas Pott. A parallel implementation for the optimization of parallel kinematic machines under process requirements. In EuCoMeS, Obergurgl (Austria), 2006.

3. Paul Bosscher and al. Cable-suspended robotic contour crafting system. Automation in Construction, 17(1):45 - 55, 2007.

4. D. Chablat, Ph. Wenger, F. Majou, and J. P. Merlet. An interval analysis based study for the design and the comparison of three-degrees-of-freedom parallel kinematic machines. The International Journal of Robotics Research, 23(6):615-624, 2004.

5. M. Gouttefarde, J.-P. Merlet, and D. Daney. Determination of the wrench-closure workspace of 6-dof parallel cable-driven mechanisms. In Jadran Lennarcic and B. Roth, editors, Advances in Robot Kinematics, pages 315-322. Springer Netherlands, 2006.

6. R. Horst and H. Tuy. Global Optimization: Deterministic Approaches. Springer, 1996.

7. H. M. Irvine. Cable Structures. MIT Press, 1981.

8. L. Jaulin, M. Kieffer, O. Didrit, and E. Walter. Applied Interval Analysis. Springer, 2001.

9. J. P. Merlet. Solving the forward kinematics of a gough-type parallel manipulator with interval analysis. The international Journal of Robotics Research, 23(3):221-235, 2004.

10. Jean-Pierre Merlet. Parallel Robots, 2nd Edition, volume 128. Springer, 2006.

11. A Ming and T Higuchi. Study On Multiple Degree-Of-Freedom Positioning Mechanism Using Wires .2. Development Of A Planar Completely Restrained Positioning Mechanism. International Journal Of The Japan Society For Precision Engineering, 28(3):235-242, Sep 1994.

12. R. E Moore. Interval Analysis. Prentice-Hall, 1966.

13. A. Neumaier. Interval Methods for Systems of Equations. Cambridge Univ. Press, 1990.

14. M. Saeed Varziri and Leila Notash. Kinematic calibration of a wire-actuated parallel robot. Mechanism and Machine Theory, 42(8):960 - 976, 2007. 